\title{
EDITORIAL
}

\section{Hyperkalemia: a threat to RAAS inhibition?}

T

he renin-angiotensin-aldosterone system (RAAS) has a pathogenetic role in several edematous disorders, including cardiac disease, liver disease, drugresistant hypertension, chronic kidney disease (CKD), the metabolic syndrome, and diabetes mellitus (Schrier, R. W. et al. Clin. J. Am. Soc. Nephrol. in press). The finding that angiotensin II and aldosterone are proinflammatory, profibrotic, and can cause oxidative injury, has led to the development of several agents that inhibit the RAAS. These agents include angiotensin-convertingenzyme (ACE) inhibitors, angiotensin-receptor blockers (ARBs), mineralocorticoid-receptor antagonists, and direct renin inhibitors, all of which can induce hyperkalemia as an adverse effect. A reasonable definition of hyperkalemia is a serum potassium concentration of $\geq 5.5 \mathrm{mmol} / \mathrm{l}$. When using inhibitors of the RAAS, physicians must be aware of clinical circumstances that may promote the development of hyperkalemia. For example, use of concomitant therapies, such as $\beta$-blockers, nonsteroidal anti-inflammatory agents, potassium-sparing diuretics, heparin, and calcineurin inhibitors, or the absence of a low-potassium diet, are well recognized to cause further increases in the plasma concentration of potassium in patients on RAAS inhibitors-a fact that is sometimes forgotten.

Use of mineralocorticoid-receptor antagonists has been shown to benefit patients with cardiac and liver disease. In the Randomized Aldosterone Evaluation Study (RALES), administration of the mineralocorticoid-receptor antagonist spironolactone ( $25 \mathrm{mg}$ daily) decreased the risk of mortality in patients with advanced heart failure (Pitt, B. et al. N. Engl. J. Med. 341, 709-717; 1999) and in the Eplerenone Post-Acute Myocardial Infarction Heart Failure Efficacy and Survival Study (EPHESUS), eplerenone (50 mg daily) reduced the risk of mortality associated with acute myocardial infarction (Pitt, B. et al. Circulation 118, 1643-1650; 2008). The effects of mineralocorticoid-receptor antagonists in RALES and EPHESUS were largely independent of natriuretic effects, with only a $0.3-0.4 \mathrm{mmol} / 1$ rise in serum potassium concentration associated with mineralocorticoidreceptor antagonism. An observational study that reported an increase in the rates of hospitalization and in-hospital deaths associated with hyperkalemia after the publication of RALES (Juurlink, D. et al. N. Engl. J. Med. $351,543-551 ; 2004)$ has had a role in limiting the use of natriuretic doses (50-100 mg per day) of spironolactone in treating sodium retention in patients with advanced heart failure, despite the study's limitations and lack of confirmation of the study's findings. This effect has also occurred in spite of the evidence of secondary hyperaldosteronism in patients with advanced heart failure and the finding from the ADHERE study that nearly $50 \%$ of hospitalized patients with decompensated heart failure are discharged with no improvement in symptoms of congestion (Fonarow, G. C. et al. Arch. Intern. Med. 165, 1469-1477; 2005). By contrast, in patients with decompensated cirrhosis and ascites, natriuretic doses of spironolactone are the primary diuretic of choice because of the association of this entity with secondary hyperaldosteronism.

In patients with decompensated cirrhosis, diuretic resistance is defined as no change in urinary sodium excretion after administration of $400 \mathrm{mg}$ of spironolactone and $160 \mathrm{mg}$ of furosemide. The kaliuretic effect of loop diuretics such as furosemide means that hyperkalemia associated with mineralocorticoid-receptor antagonists can generally be avoided by coadministration of furosemide, particularly if the patient is on a potassium-restricted diet.

Modest doses of a mineralocorticoid-receptor antagonist (for example, $25 \mathrm{mg}$ per day of spironolactone) have been shown to effectively lower blood pressure in patients with multidrug-resistant hypertension who are already receiving an ACE inhibitor, often in combination with a diuretic. In some studies, the addition of a mineralocorticoid-receptor antagonist to ACE inhibitor therapy has been shown to be more effective than the addition of an ARB to ACE inhibitor therapy in treating multidrug-resistant hypertension. The incidence of hyperkalemia in patients with normal renal function who are treated with dual RAAS inhibitors in the absence of other potassium-retaining therapies seems to be approximately $5 \%$. The degree to which blood pressure is lowered in patients with multidrug-resistant hypertension after the addition of a mineralocorticoid-receptor antagonist to standard therapy seems to be comparable to the degree of blood pressure lowering observed with such treatment in patients with primary hyperaldosteronism.

RAAS inhibition in patients with CKD, particularly in those with diabetic nephropathy, decreases urinary excretion of protein and/or albumin-a harbinger of decreased renal deterioration. In patients with diabetes and proteinuria, therapy with an ACE inhibitor or an ARB slows progression of renal disease. A 2009 study in diabetic patients indicated that predictors of hyperkalemia in patients with diabetic nephropathy on RAAS-inhibiting therapy were a baseline plasma potassium concentration $>4.5 \mathrm{mmol} / \mathrm{l}$ and/or an estimated glomerular filtration rate $<45 \mathrm{ml} / \mathrm{min}$ (Khosla, N. et al. Am. J. Nephrol. 30, 418-424; 2009). It should be emphasized, however, that patients in that study were

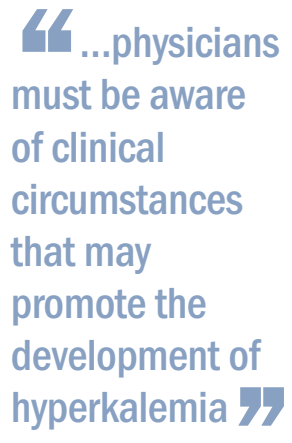

Robert W. Schrier

is the Editor-in-Chief of Nature Reviews Nephrology.

Competing interests R. W. Schrier declares an association with the following company: Otsuka

Pharmaceuticals. See the article online for full details of the relationship. 
receiving potassium-losing diuretics. An extensive study of patients with CKD or heart failure who were treated with RAAS inhibitors revealed an incidence of hyperkalemia of $5-10 \%$ (Weir, M. R. \& Rolfe, M. Clin. J. Am. Soc. Nephrol. 5, 531-548; 2010).

The role of the RAAS in the worldwide obesitydiabetes epidemic is gaining increasing recognition. Obesity per se is known to stimulate the RAAS. Moreover, evidence suggests that adipocytes release secretagogues that stimulate aldosterone production independent of the renin-angiotensin system. Mineralocorticoid receptors are present in several tissues in addition to the kidney, including the heart, blood vessels, and brain. Thus, mineralocorticoid-receptor antagonists may exert beneficial anti-inflammatory and antifibrotic effects in various tissues in patients with obesity, the metabolic syndrome, and/or diabetes mellitus. Little information exists about the incidence of hyperkalemia associated with use of mineralocorticoid-receptor antagonists in these settings. In general, however, the incidence of hyperkalemia with RAAS-inhibition monotherapy is
$<2 \%$, whereas with dual RAAS inhibition the incidence increases to $\sim 5 \%$.

Acute treatments for hyperkalemia that is associated with electrocardiogram changes are well known, and include insulin and glucose, $\beta$-agonists, calcium and, if necessary, dialysis. Of note, however, the concomitant administration of the ion-exchange resin sodium polystyrene sulfonate (Kayexalate ${ }^{\mathrm{TM}}$, Sanofi-Aventis, Delaware, NJ) with sorbitol for the treatment of hyperkalemia may be associated with ischemic colitis. Moreover, the efficacy of this combination in lowering plasma potassium concentration has recently been challenged (Sterns, R. H. et al. J. Am. Soc. Nephrol. doi:10.1681/ASN.2010010079).

Thus, the importance of RAAS inhibition has emerged in several clinical disorders. The careful monitoring of plasma potassium concentration during such therapy, however, is indicated.

doi:10.1038/nrneph.2010.52 